\title{
Convective Heat Transfer of Unsteady Pulsed Flow in Sinusoidal Constricted Tube
}

\author{
J. Batina ${ }^{1}$, S. Blancher ${ }^{1}$, C. Amrouche ${ }^{2}$, M. Batchi ${ }^{2}$ and R. Creff ${ }^{1}$ \\ ${ }^{1}$ Laboratoire des Sciences de l'Ingénieur Appliquées à la Mécanique et l'Electricité \\ Université de Pau et des Pays de l'Adour, Avenue de l'Université - 64000 Pau; \\ ${ }^{2}$ Laboratoire de Mathématiques Appliquées- CNRS UMR 5142 \\ Université de Pau et des Pays de l'Adour, Avenue de l'Université - 64000 Pau;
}

France

\section{Introduction}

In many industrial engineering and other technological processes, it is crucial to characterise heat and mass transfer, for example to avoid thermo mechanical damages.

Particularly, in the inlet region of internal pulsed flows, unsteady dynamic and thermal effects can present large amplitudes. These effects are mainly located in the wall region. This suggests the existence of intense unsteady stresses at the wall (shear, friction or thermal stresses). Our studies (André et al., 1987; Batina, 1995; Creff et al., 1985) show that there could exist an 'adequacy' of different parameters such as Reynolds or Prandtl numbers, leading to large amplitudes for the unsteady velocity and temperature in the entry zone if compared to those encountered downstream in the fully developed region. Consequently, in order to obtain convective heat transfer enhancement, most of the studies are linked to:

- Firstly, the search for optimal geometries (undulated or grooved channels, tube with periodic sections, etc.) : among those geometrical studies, one can quote the investigations of Blancher, 1991; Ghaddar et al., 1986, for the wavy or grooved plane geometries, in order to highlight the influence of the forced or natural disturbances on heat transfer.

- Secondly, the search for particular flow conditions (transient regime, pulsed flow, etc.): for example those linked to the periodicity of the pressure gradient (Batina, 1995; Batina et al. 2009; Chakravarty \& Sannigrahi, 1999; Hemida et al., 2002), or those which impose a periodic velocity condition (Lee et al., 1999; Young Kim et al., 1998) or those which carry on time periodic deformable walls.

The main objective of this study is to analyse the special case of convective heat transfer of an unsteady pulsed, laminar, incompressible flow in axisymmetric tubes with periodic sections. The flow is supposed to be developing dynamically and thermally from the duct inlet. The wall is heated at constant and uniform temperature.

One of the originality of this study is the choice of Chebyshev polynomials basis in both axial and radial directions for spectral methods, the use of spectral collocation method and the introduction of a shift operator to satisfy non homogeneous boundary conditions for spectral Galerkin formulation. A comparison of results obtained by the two spectral methods is given. A Crank - Nicolson scheme permits the resolution in time. 


\subsection{Nomenclature}

\begin{tabular}{|c|c|c|}
\hline$a$ & thermal diffusity $\left[\mathrm{m}^{2} / \mathrm{s}\right]$ & dimensionless total wavelength \\
\hline $\mathrm{e}$ & reduced amplitude & dimensionless temperature: \\
\hline$h$ & wall function & $\theta=\left(T-T_{\infty}\right) /\left(T_{W}-T_{\infty}\right)$ \\
\hline $\mathrm{H}$ & periodic sinusoidal radius $[\mathrm{m}]$ & $\mu \quad$ dynamic viscosity $\left[\mathrm{Ns} / \mathrm{m}^{2}\right]$ \\
\hline$L$ & geometric half-length tube $[\mathrm{m}]$ & $v=\mu / \rho$ kinematic viscosity: $\left[\mathrm{m}^{2} / \mathrm{s}\right]$ \\
\hline$R$ & tube radius at the constriction $[\mathrm{m}]$ & $\rho \quad$ fluid density $\left[\mathrm{Kg} / \mathrm{m}^{3}\right]$ \\
\hline$r$ & radial co-ordinate $[\mathrm{m}]$ & modulation flow rate \\
\hline$T$ & fluid temperature $[K]$ & vorticity function $[1 / s]$ \\
\hline$T_{\infty}$ & duct inlet temperature $[K]$ & $\psi \quad$ stream function $\left[\mathrm{m}^{3} / \mathrm{s}\right]$ \\
\hline$t$ & time $[s]$ & $\Omega \quad$ pulsation $[\mathrm{rad} / \mathrm{s}]$ \\
\hline$u$ & axial velocity $[\mathrm{m} / \mathrm{s}]$ & Dimensionless numbers \\
\hline $\bar{u}_{0}$ & mean bulk velocity $[\mathrm{m} / \mathrm{s}]$ & Reynolds number: $R e=R \bar{u}_{0} / \nu$ \\
\hline$v$ & radial velocity $[\mathrm{m} / \mathrm{s}]$ & Prandtl number: $P r=v / a$ \\
\hline & axial co-ordinate $[\mathrm{m}]$ & Nusselt number \\
\hline \multicolumn{2}{|c|}{ Greek symbols } & $\theta_{0 \mathrm{~m}}(x)$ averaged bulk temperature \\
\hline \multicolumn{2}{|c|}{$\Phi_{W}$ wall heat flux $\left[\mathrm{W} / \mathrm{m}^{2}\right]$} & 0 steady flow; $\mathrm{W}$ : wall \\
\hline
\end{tabular}

\subsection{Suggested keywords}

Convective heat transfer - sinusoidal constricted tube - axisymmetric geometry - pulsed laminar, incompressible flow - spectral collocation method - Chebyshev-Gauss-Lobatto mesh - spectral Galerkin formulation - shift operator method - Crank - Nicolson resolution in time.

\section{General hypothesis and governing equations}

\subsection{General hypothesis}

We consider a Newtonian incompressible fluid flow developing inside an axisymmetric cylindrical duct with periodic sinusoidal radius. The unsteadiness imposed to the flow corresponds to a source of periodic pulsations generating plane waves. This flow is described in terms of an unsteady pulsed flow superimposed on a steady one, without reverse flow at the entry and the exit sections. With regard to the thermal problem, the wall is heated at constant and uniform temperature, and the fluid inlet temperature is equal to 
the upstream ambient temperature. Physical constants are supposed to be independent of the temperature, which involves that the motion and energy equations are uncoupled.

\subsection{Governing equations}

With the 2D hypothesis, we use the vorticity-stream function formulation $(\omega, \psi)$ for the Navier-Stokes equations in which the incompressibility condition is automatically satisfied. In fact, the essential advantage of this formulation compared to the primitive variables (velocity-pressure formulation) is the reduction of the number of unknown functions and the non-used of the pressure. On the other hand, Navier-Stokes equations become a fourth order Partial Differential Equations whose expressions in cylindrical coordinates are:

$$
\frac{\partial \hat{\omega}}{\partial t}-\frac{1}{r} \frac{\partial \psi}{\partial z} \frac{\partial \hat{\omega}}{\partial r}+\frac{1}{r} \frac{\partial \psi}{\partial r} \frac{\partial \hat{\omega}}{\partial z}+\frac{2}{r^{2}} \frac{\partial \psi}{\partial z} \hat{\omega}=v\left(\frac{\partial^{2} \hat{\omega}}{\partial r^{2}}+\frac{\partial^{2} \hat{\omega}}{\partial z^{2}}-\frac{1}{r} \frac{\partial \hat{\omega}}{\partial r}\right)=v \Delta \hat{\omega}
$$

It is important to note that we have only one unknown function, i.e.: $\psi$. The vorticity function $\omega$ is linked to $\psi$ by the relation:

$$
\hat{\omega}=r \omega=-\left(\frac{\partial^{2} \psi}{\partial r^{2}}+\frac{\partial^{2} \psi}{\partial z^{2}}-\frac{1}{r} \frac{\partial \psi}{\partial r}\right)=-\Delta \psi
$$

Velocity components are given by:

$$
u=\frac{1}{r} \frac{\partial \psi}{\partial r} \text { and } v=-\frac{1}{r} \frac{\partial \psi}{\partial z}
$$

The energy equation is:

$$
\left(\frac{\partial T}{\partial t}+u \frac{\partial T}{\partial z}+v \frac{\partial T}{\partial r}\right)=a\left(\frac{\partial^{2} T}{\partial r^{2}}+\frac{\partial^{2} T}{\partial z^{2}}+\frac{1}{r} \frac{\partial T}{\partial r}\right)
$$

\section{Boundary conditions}

The present problem is unsteady. This unsteadiness is generated at the initial instant $t=0$, and is sustained during all the time by a source of upstream pulsations. For both steady and unsteady flow, the following boundary conditions are available for any time $t \geq 0$ :

- Entry: for the thermal problem, the inlet fluid temperature is equal to the upstream ambient temperature: $T=T_{\infty}$.

- Exit: the flow velocity is normal to the exit section and verifies the classical condition:

$$
v=0 \text { and } \frac{\partial T}{\partial z}=\frac{\partial u}{\partial z}=0 .
$$

- Axis: the flow preserves at each time an axial symmetry:

$$
\frac{\partial u}{\partial r}=v=\frac{\partial T}{\partial r}=0
$$

- Wall: no slip condition is imposed and the wall is heated at constant temperature: 


$$
u=v=0 ; T=T_{W} .
$$

For dynamic conditions at the entry section, we impose:

- Steady flow ( $t=0$ time step)

- Entry: for the dynamic problem, Poiseuille profile boundary condition is chosen

$$
u(z=0, r)=2 \bar{u}_{0}\left(1-\left(\frac{r}{R}\right)^{2}\right)
$$

- Unsteady flow $(t>0)$

- Entry: the source of imposes a periodic pressure gradient modulation. Then the velocity axial component and the stream function $\psi$ have a Fourier series expansion in time:

$$
f(z=0, r, t)=f_{0}(z=0, r)\left(1+\sum_{n=1}^{N_{F}} \tau^{n} \cdot \sin (n \Omega t)\right)
$$

where $f$ represents $u$ or $\psi$. At this section, to avoid reverse flow, we impose: $\tau<1$.

\section{New formulation and resolution of the dynamic and thermal problem}

\subsection{New formulation of the dynamic problem}

\subsubsection{Dimensionless quantities and variables transformations}

One chooses for dimensionless variables:

$$
\tilde{r}=\frac{r}{R} ; \tilde{z}=\frac{z}{R} ; \tilde{t}=\frac{t}{\bar{t}_{0}} ; \tilde{\omega}=\frac{\hat{\omega}}{\bar{\omega}_{0}} ; \tilde{\psi}=\frac{\psi}{\bar{\psi}_{0}} ; \tilde{u}=\frac{u}{\bar{u}_{0}} ; \tilde{\mathrm{v}}=\frac{v}{u_{0}}
$$

with

$$
\bar{t}_{0}=\frac{\mathrm{L}}{\overline{\mathrm{u}}_{0}} ; \bar{\omega}_{0}=\frac{\bar{u}_{0}}{R} ; \bar{\psi}_{0}=\bar{u}_{0} R^{2}
$$

The Reynolds number $R e$ is based on the radius at the duct constriction:

$$
\operatorname{Re}=R \bar{u}_{0} / v
$$

In order to obtain a computational square domain permitting the use of two dimensional Chebyshev polynomials, we proceed to a space variables transformation. This one is inspired by Sobey, 1980, and modified by Blancher, 1991. It has been adapted to the axisymmetric geometry used in this study. Afterwards, we note by $H(z)$ the duct periodic radius. Then we define:

$$
\rho=\frac{\tilde{r}}{h(x)} ; x=\frac{\tilde{z}}{\lambda}-1
$$

with

$$
\lambda=\frac{L}{R} ; h(x)=\frac{1}{R} H[(x+1) \cdot L]
$$


and (see equation 73)

$$
H(z)=R\left\{1+\frac{e}{2}\left[1-\cos \left(\pi \cdot n_{O} \frac{z}{L}\right)\right]\right\} \Leftrightarrow h(x)=1+\frac{e}{2}\left[1-\cos \left(\pi \cdot n_{O}(x+1)\right)\right]
$$

Finally, the study domain is transformed into a rectangle $-1 \leq x \leq 1$ and $0 \leq \rho \leq 1$ representing the half - space of the square: $[-1,1] \times[-1,1]$.

\subsubsection{New system of unsteady dynamic governing equations}

Considering the transformation of variables defined before, the new stream - vorticity formulation of this problem is:

$$
\left\{\begin{array}{l}
\breve{\omega}=-\Delta_{f} \tilde{\psi} \\
h^{2} \frac{\partial \breve{\omega}}{\partial \tilde{t}}+\frac{1}{\rho}\left(\frac{\partial \tilde{\psi}}{\partial \rho} \frac{\partial \breve{\omega}}{\partial x}-\frac{\partial \tilde{\psi}}{\partial x} \frac{\partial \breve{\omega}}{\partial \rho}\right)+\frac{2}{\rho^{2}}\left(\frac{\partial \tilde{\psi}}{\partial x}-2 \rho \frac{h^{\prime}}{h} \frac{\partial \tilde{\psi}}{\partial \rho}\right) \breve{\omega}=\frac{1}{\widehat{\operatorname{Re}}} \Delta_{g} \breve{\omega}
\end{array}\right.
$$

where:

$$
\Delta_{f} \tilde{\psi}=\left\{h^{2} \frac{\partial^{2} \tilde{\psi}}{\partial x^{2}}-2 \rho h^{\prime} h \frac{\partial^{2} \tilde{\psi}}{\partial x \partial \rho}+\left[\lambda^{2}+\rho^{2} h^{\prime 2}\right] \frac{\partial^{2} \tilde{\psi}}{\partial \rho^{2}}+\left[\rho\left(2 h^{\prime 2}-h h^{\prime \prime}\right)-\frac{\lambda^{2}}{\rho}\right] \frac{\partial \tilde{\psi}}{\partial \rho}\right\}
$$

and

$$
\left\{\begin{array}{l}
\breve{\omega}=\lambda^{2} h^{2} \tilde{\omega} \\
\Delta_{g} \breve{\omega}=-\left[\begin{array}{l}
A_{g}(x, \rho) \frac{\partial^{2} \breve{\omega}}{\partial x^{2}}+B_{g}(x, \rho) \frac{\partial^{2} \breve{\omega}}{\partial \rho^{2}}+C_{g}(x, \rho) \frac{\partial \breve{\omega}}{\partial \rho} \\
+D_{g}(x, \rho) \frac{\partial^{2} \breve{\omega}}{\partial x \partial \rho}+E_{g}(x, \rho) \frac{\partial^{2} \breve{\omega}}{\partial x}+F_{g}(x, \rho) \breve{\omega}
\end{array}\right]
\end{array}\right.
$$

with:

$$
\begin{aligned}
& \left\{\begin{array}{l}
A_{g}(x, \rho)=h^{2} ; B_{g}(x, \rho)=\lambda^{2}+\rho^{2} h^{\prime 2} ; C_{g}(x, \rho)=\rho\left(6 h^{\prime 2}-h h^{\prime \prime}\right)-\frac{\lambda^{2}}{\rho} \\
D_{g}(x, \rho)=-2 \rho h h^{\prime} ; E_{g}(x, \rho)=-4 h h^{\prime} ; F_{g}(x, \rho)=2\left(3 h^{\prime 2}-h h^{\prime \prime}\right)
\end{array}\right. \\
& \qquad \hat{\operatorname{Re}}=\tilde{\operatorname{Re}} \lambda^{2}=\operatorname{Re} \lambda \\
& \Delta_{f} \tilde{\psi}=\left\{h^{2} \frac{\partial^{2} \tilde{\psi}}{\partial x^{2}}-2 \rho h^{\prime} h \frac{\partial^{2} \tilde{\psi}}{\partial x \partial \rho}+\left[\lambda^{2}+\rho^{2} h^{\prime 2}\right] \frac{\partial^{2} \tilde{\psi}}{\partial \rho^{2}}+\left[\rho\left(2 h^{\prime 2}-h h^{\prime \prime}\right)-\frac{\lambda^{2}}{\rho}\right] \frac{\partial \tilde{\psi}}{\partial \rho}\right\}
\end{aligned}
$$

\subsubsection{The dynamic steady problem formulation}

The dynamic steady problem corresponding to problem (16) is written as follows: 


$$
\left\{\begin{array}{l}
\breve{\omega}=-\Delta_{f} \tilde{\psi} \\
\frac{1}{\rho}\left(\frac{\partial \tilde{\psi}}{\partial \rho} \frac{\partial \breve{\omega}}{\partial x}-\frac{\partial \tilde{\psi}}{\partial x} \frac{\partial \breve{\omega}}{\partial \rho}\right)+\frac{2}{\rho^{2}}\left(\frac{\partial \tilde{\psi}}{\partial x}-2 \rho \frac{h^{\prime}}{h} \frac{\partial \tilde{\psi}}{\partial r}\right) \breve{\omega}=\frac{1}{\hat{\operatorname{Re}}} \Delta_{g} \breve{\omega}
\end{array}\right.
$$

Important: for reason of convenience, the radius $\rho$ will be noted $r$.

\subsection{New formulation of the thermal problem}

For the thermal problem, the temperature $\tilde{\theta}$ is made dimensionless in a classic way:

$$
\tilde{\theta}=\frac{T-T_{\infty}}{T_{W}-T_{\infty}}
$$

\subsubsection{The thermal unsteady problem formulation}

Using (1) and (10)-(15), the dimensionless energy equation can be written as follows:

$$
h^{2} \frac{\partial \tilde{\theta}}{\partial \tilde{t}}+h^{2} \tilde{u} \frac{\partial \tilde{\theta}}{\partial x}+h\left(\tilde{\mathrm{v}} \lambda-\tilde{u} r h^{\prime}\right) \frac{\partial \tilde{\theta}}{\partial r}=\frac{1}{\widehat{\operatorname{RePr}}} \Delta_{f} \tilde{\theta}
$$

with:

$$
\Delta_{f} \tilde{\theta}=h^{2} \frac{\partial^{2} \tilde{\theta}}{\partial x^{2}}-2 r h^{\prime} h \frac{\partial^{2} \tilde{\theta}}{\partial x \partial r}+\left[\lambda^{2}+r^{2} h^{\prime 2}\right] \frac{\partial^{2} \tilde{\theta}}{\partial r^{2}}+\left[r\left(2 h^{\prime 2}-h h^{\prime \prime}\right)+\frac{\lambda^{2}}{r}\right] \frac{\partial \tilde{\theta}}{\partial r}
$$

\subsubsection{The thermal steady problem formulation}

The dimensionless steady state energy problem related to the equation (24) is:

$$
h^{2} \tilde{u} \frac{\partial \tilde{\theta}}{\partial x}+h\left(\tilde{v} \lambda-\tilde{u} r h^{\prime}\right) \frac{\partial \tilde{\theta}}{\partial r}=\frac{1}{\widehat{\operatorname{RePr}}} \Delta_{f} \tilde{\theta}
$$

\section{Numerical resolution using spectral methods}

\subsection{Trial functions and development orders}

The spectral methods consist in projecting any unknown function $f(x, r, t)$ on trial functions as follows:

$$
f(x, r, t)=\sum_{k=0}^{N_{x}} \sum_{1=0}^{N_{r}} f_{k l}(t) P_{l}(r) Q_{k}(x)
$$

where $N_{x}$ and $N_{r}$ are the development orders according to the axis $x$ and $r$ respectively. The basis functions $P_{l}(r)$ and $Q_{k}(x)$ are generally trigonometric or polynomial functions (Chebyshev, Legendre, etc.) according to different boundary conditions situations. The time dependant coefficients $f_{k l}(t)$ are the unknowns of the problem. For our problem, the function $f$ represents $\breve{\omega}, \tilde{\psi}$ or $\tilde{\theta}$. For a steady problem, the coefficients $f_{k l}(t)$ are time independent. 
It is necessary to study the influence of the physical parameters such as the Reynolds number to remain in 2D hypothesis. From a numerical point of view we will show the influence of the polynomials degrees particularly for the thermal problem.

\subsection{The choice of basis functions}

Because no symmetry condition is imposed at the boundaries of our half-domain of study, we choose basis functions constructed from Chebyshev polynomials (Bernardi \& Maday, 1992; Canuto et al., 1988) instead of trigonometric trial functions. Then, $P_{l}(r)$ and $Q_{k}(x)$ are written as linear combination of Chebyshev polynomials. Their expressions depend on the boundary conditions and the spectral method used (Galerkin or collocation method).

Generally, with Galerkin method, Dirichlet or Neuman boundary conditions imposed to trial functions must be homogeneous, but it is not necessary for collocation method (see Galerkin and collocation methods below).

The basis $P_{l}(r)$ and $Q_{k}(x)$ are written as a linear combination of Chebyshev polynomials such as (Gelfgat, 2004; Shen, 1994, 1995, 1997):

$$
P_{l}(r)=T_{l}(r)+\sum_{i=1}^{n} \alpha_{l} T_{l+i}(r) \text { and } Q_{k}(x)=T_{k}(x)+\sum_{i=1}^{m} \beta_{k} T_{k+i}(x)
$$

where $n$ (respectively $m$ ) is the number of boundary conditions according to the radial direction $r$ (respectively the axial direction $x$ ), and $T_{k}(x)$ is the Chebyshev polynomial of degree $k$.

\subsubsection{Advantages and limitations of spectral methods}

Spectral methods are used successfully in many problems of physics, mainly those involving periodic physical phenomena in space and / or in time. Its main advantage is its high degree of accuracy, compared with some methods such as finite differences, finite elements or finite volumes (Bernardi \& Maday, 1992; Canuto et al., 1988; Gelfgat, 2004; Shen, 1994, 1995, 1997). Spectral methods are particularly suitable to study instabilities phenomena, selfmaintained or forced, occuring in Computational Fluid Dynamics. However, spectral methods are limited to simple geometries. For complicated study domains, an alternative way may be using spectral finite elements. The second disadvantage of these methods is their cost of implementing and their high CPU calculations. The matrices obtained are usually full and strategies for solving linear or nonlinear systems remain limited.

\section{Numerical resolution of the dynamic and thermal problem using spectral galerkin formulation}

\subsection{Numerical resolution of the dynamic steady problem}

The steady dynamic problem is given by the equation (22). Generally, this problem is written with classical homogeneous boundary conditions. One of the originalities of this study is the use of a relevment function allowing the introduction of non homogeneous boundary conditions. For this reason, the unknown stream function $\tilde{\psi}(x, r)$ is written by mean of the Poiseuille stream function $\varphi_{0}(r)$ corresponding to the Poiseuille velocity imposed at the duct entry as:

$$
\tilde{\psi}(x, r)=\psi_{0}(x, r)+\varphi_{0}(r)
$$


where the stream function $\psi_{0}(x, r)$ verifies homogeneous boundary conditions in both directions $x$ and $r$.

The equation (22) becomes:

$$
\frac{1}{r^{2}} \frac{\partial \psi_{0}}{\partial x}\left(\alpha(\omega)+\alpha_{\Phi}\right)+\frac{1}{r} \frac{\partial \psi_{0}}{\partial r}\left(\beta(\omega)+\beta_{\Phi}\right)+\frac{1}{r} \frac{\partial \varphi_{0}}{\partial r} \beta(\omega)-\frac{1}{\widehat{\operatorname{Re}}} \gamma(\omega)=\frac{1}{\widehat{\operatorname{Re}}} \gamma_{\Phi}-\frac{1}{r} \frac{\partial \varphi_{0}}{\partial r} \beta_{\Phi}
$$

with:

$$
\begin{gathered}
\alpha(\omega)=2 \omega-r \frac{\partial \omega}{\partial r} ; \alpha_{\Phi}=2 \Phi-r \frac{\partial \Phi}{\partial r} ; \beta(\omega)=\frac{\partial \omega}{\partial x}-4 \frac{h^{\prime}}{h} \omega ; \beta_{\Phi}=\frac{\partial \Phi}{\partial x}-4 \frac{h^{\prime}}{h} \Phi ; \\
\gamma(\omega)=\Delta_{g} \omega ; \gamma_{\Phi}=\Delta_{g} \Phi ; \\
\Phi(x, r)=-\Delta_{f} \varphi_{0}(r) .
\end{gathered}
$$

The corresponding Galerkin method consists in projecting the discretized equations on a Chebyshev polynomials basis, taking into account the whole boundary conditions (Canuto et al., 1988). Then, according to the general formulation of spectral methods, the streamfunction $\psi_{0}$ is projected on trial functions as follows:

$$
\psi_{0}(x, r)=\sum_{k=0}^{N_{x}} \sum_{l=0}^{N_{r}} \psi_{k l} P_{2 l}(r) Q_{k}(x)
$$

Because of the symmetry property on the whole axisymmetric domain of the problem, $P_{2 l}(r)$ will be an even function. To construct the basis $P_{2 l}(r)$, we choose a linear combination of Chebyshev polynomials such as (Gelfgat, 2004; Shen, 1994, 1995, 1997):

$$
P_{2 l}(r)=T_{2 l}(r)+\sum_{i=1}^{n} \alpha_{l i} T_{2(l+i)}(r)
$$

where $n$ is the number of boundary conditions according to radial direction $r$ ( $n=3$ here, see bellow).

The coefficients $\alpha_{l i}$ are determined so that $P_{2 l}(r)$ satisfies the corresponding homogeneous boundary conditions:

$$
\begin{gathered}
\left.\frac{\partial P_{2 l}}{\partial r}=0 \text { at } r= \pm 1 \quad \text { (because } \frac{\partial \tilde{\psi}}{\partial r}=0 \text { at } r= \pm 1\right) \\
P_{2 l}(r)=0 \text { at } r= \pm 1 \quad \text { (flow-rate condition at } r= \pm 1 \text { ) } \\
P_{2 l}(r)=0 \text { at } r=0 \text { (axial symmetry) }
\end{gathered}
$$

So, one can determine all coefficients $\alpha_{l i}$. Finally we have:

$$
P_{2 l}(r)=T_{2 l}(r)-\frac{l+1}{l+2} T_{2(l+1)}(r)-T_{2(l+2)}(r)+\frac{l+1}{l+2} T_{2(l+3)}(r)
$$

A similar analysis is available for the choice of $Q_{k}(x)$ basis functions: 


$$
Q_{k}(x)=T_{k}(x)+\sum_{i=1}^{m} \beta_{k i} T_{(k+i)}(x)
$$

where $m=3$ here (see bellow). The velocity boundary conditions imply that the stream function must satisfy the corresponding homogeneous boundary conditions as:

$$
\begin{gathered}
Q_{k}^{\prime}(-1)=0 \text { at } \mathrm{x}=-1(\tilde{v}=0 \text { at } x=-1) \\
\left.Q_{k}(-1)=0 \text { at } \mathrm{x}=-1 \text { (Poiseuille profile } x=-1\right) \\
Q_{k}^{\prime}(1)=0 \text { at } \mathrm{x}=1 \quad(\tilde{v}=0 \quad \text { at } x=1)
\end{gathered}
$$

Finally we obtain:

$$
Q_{k}(x)=T_{k}(x)-\frac{(k+3)^{2}(k+1)}{(k+2)^{2}(k+2)} T_{k+1}(x)-\frac{k^{2}}{(k+2)^{2}} T_{k+2}(x)+\frac{(k+3)^{2}(k+1)}{(k+2)^{2}(k+2)} T_{k+3}(x)
$$

Let us define the Chebyshev scalar product as:

$$
(\psi, \phi)=\iint_{\Delta} \psi(x, r) \phi(x, r)\left(\frac{1}{\sqrt{1-x^{2}}} \cdot \frac{1}{\sqrt{1-r^{2}}}\right) d x d r
$$

where $\Delta$ is the square: $\Delta=[-1,1] \times[-1,1]$.

Taking as test function:

$$
\phi(x, r)=Q_{k}(x) P_{2 l}(r), \text { for } 0 \leq \mathrm{k} \leq \mathrm{N}_{\mathrm{x}}, 0 \leq 1 \leq \mathrm{N}_{\mathrm{r}}
$$

the Galerkin spectral method consist to make scalar products between the non linear equation (30) and each test function $Q_{i}(x) P_{2 j}(r)$, by writing:

$$
\begin{aligned}
& \left(\frac{1}{r^{2}} \frac{\partial \psi_{0}}{\partial x}\left(\alpha(\omega)+\alpha_{\Phi}\right)+\frac{1}{r} \frac{\partial \psi_{0}}{\partial r}\left(\beta(\omega)+\beta_{\Phi}\right)+\frac{1}{r} \frac{\partial \varphi_{0}}{\partial r} \beta(\omega)-\frac{1}{\widehat{\operatorname{Re}}} \gamma(\omega), Q_{i}(x) P_{2 j}(r)\right)= \\
& =\left(\frac{1}{\widehat{\operatorname{Re}}} \gamma_{\Phi}-\frac{1}{r} \frac{\partial \varphi_{0}}{\partial r} \beta_{\Phi}, Q_{i}(x) P_{2 j}(r)\right)
\end{aligned}
$$

Finally, we obtain a system of $N_{x r}=\left(N_{x}+1\right)\left(N_{r}+1\right)$ non linear equations with $N_{x r}$ unknowns, solved by Newton algorithm.

\subsection{Numerical resolution of the dynamic unsteady problem}

From equation (16), introducing the unknown $\psi$ function such as:

$$
\tilde{\psi}(x, r, t)=\psi(x, r, t)+\varphi(r) A(t)
$$

and using the equations (46), we define the operator in which the unknown coefficients depend now on time:

$$
L_{\psi}(x, r, t)=-\left(\frac{1}{r^{2}} \frac{\partial \psi}{\partial x}\left(\alpha(\omega)+\alpha_{\Phi}\right)+\frac{1}{r} \frac{\partial \psi}{\partial r}\left(\beta(\omega)+\beta_{\Phi}\right)+\frac{1}{r} \frac{\partial \varphi}{\partial r} \beta(\omega)-\frac{1}{\hat{\operatorname{Re}}} \gamma(\omega)\right)+\frac{1}{\hat{\operatorname{Re}}} \gamma_{\Phi}-\frac{1}{r} \frac{\partial \varphi}{\partial r} \beta_{\Phi}(48)
$$


Then the previous problem (16) can take the following form:

$$
h^{2} \frac{\partial \breve{\omega}}{\partial t}=L_{\psi}(x, r, t) \text { where } \breve{\omega}=\omega+\omega_{\Phi}
$$

The operator $L_{\psi}(x, r, t)$ is nonlinear. Notice that $\omega_{\Phi}$ is the contribution coming from Poiseuille extension. The temporal discretization of (49) is made by using the $\varepsilon$-method, reduced here to Crank - Nicolson method. The advantage of this method is to be unconditionally stable. It leads to the equation below with $\varepsilon=1 / 2$, which corresponds to a two order scheme:

$$
\left\{\begin{array}{l}
h^{2} \frac{\omega^{n+1}-\omega^{n}}{\Delta t}+h^{2} \frac{\partial \omega_{\Phi}(x, r, t)}{\partial t}=\varepsilon L_{\psi^{n+1}}(x, r, t)+(1-\varepsilon) L_{\psi^{n}}(x, r, t) \\
\omega^{n}=-\Delta_{f} \psi^{n}, \forall n
\end{array}\right.
$$

where the initial condition is given by the solution of the steady problem.

The unknowns $\psi_{k l}(t)$ are obtained by solving with Newton algorithm, at each time step, the non linear system obtained with scalar products between relation (50) and test functions $Q_{i}(x) P_{2 j}(r)$, as in equation (46).

\subsection{Numerical resolution of the thermal unsteady problem}

6.3.1 Choices of the basis functions

The dimensionless energy equation is given by (25) and (25). The choice of the temperature basis functions is made in the same way as in the dynamic problem. In order to apply the Galerkin method, we consider the boundary conditions (heading 3) for the temperature $\theta$. Let us set:

$$
\tilde{\theta}(x, r, t)=\theta(x, r, t)+\theta_{R}(r)
$$

where $\theta$ is the solution satisfying the homogeneous boundary conditions and $\theta_{R}(r)$ is a smoothed gap temperature imposed at the entry. The homogeneous temperature $\theta$, truncated at development orders $M_{x}$ according to the axis $x$ and $M_{r}$ according to the radius $r$, is projected on the trial functions as follows:

$$
\theta(x, r, t)=\sum_{k=0}^{M_{x}} \sum_{1=0}^{M_{r}} \theta_{k l}(t) q_{k}(x) p_{2 l}(r)
$$

where $p_{2 l}(r)$ and $q_{k}(x)$ are built from Chebyshev polynomials as in heading 5 . According to temperature boundary conditions (heading 3 ), we obtain, at last:

$$
q_{k}(x)=T_{k}(x)+\frac{4(k+1)}{(k+1)^{2}+(k+2)^{2}} T_{k+1}(x)-\frac{(k+1)^{2}+k^{2}}{(k+1)^{2}+(k+2)^{2}} T_{k+2}(x), \quad \text { if } 0 \leq k \leq M_{x}
$$

The polynomial $p_{2 l}(r)$ is given by:

$$
p_{2 l}(r)=T_{2 l}(r)-T_{2(l+1)}(r), \quad \text { if } 0 \leq l \leq M_{r}
$$




\subsubsection{Resolution of the steady energy equation}

With $(24)$ and $\tilde{\theta}(x, r)=\theta(x, r)+\theta_{R}(r)$, the steady thermal problem is written as follows:

$$
\frac{1}{r} \frac{\partial \psi}{\partial r} \cdot \frac{\partial \theta}{\partial x}-\frac{1}{r} \frac{\partial \psi}{\partial x} \cdot \frac{\partial \theta}{\partial r}+2\left(1-r^{2}\right) \frac{\partial \theta}{\partial x}-\frac{1}{\widehat{\operatorname{RePr}}} \Delta_{f} \theta=\frac{1}{r} \frac{\partial \psi}{\partial x} \cdot \frac{\partial \theta_{R}}{\partial r}+\frac{1}{\widehat{\operatorname{RePr}}} \Delta_{f} \theta_{R}
$$

This problem is discretized by Galerkin spectral method explained above. The linear system obtained is solved by a Gauss type classical method.

\subsubsection{Resolution of the unsteady energy equation}

The unsteady problem is written as follows:

$$
\begin{aligned}
& \frac{\partial \theta}{\partial t}=-\frac{1}{h^{2}}\left(\frac{1}{r} \frac{\partial \psi}{\partial r} \cdot \frac{\partial \theta}{\partial x}-\frac{1}{r} \frac{\partial \psi}{\partial x} \cdot \frac{\partial \theta}{\partial r}+\frac{1}{r} \frac{\partial \varphi}{\partial r} \frac{\partial \theta}{\partial x}-\frac{1}{\widehat{\operatorname{RePr}}} \Delta_{f} \theta\right)+\frac{1}{h^{2}}\left(\frac{1}{r} \frac{\partial \psi}{\partial x} \cdot \frac{\partial \theta_{R}}{\partial r}+\frac{1}{\widehat{\operatorname{Re} P r}} \Delta_{f} \theta_{R}\right) \\
& \theta(x, r, 0)=\theta_{0}(x, r)
\end{aligned}
$$

where $\theta_{0}(x, r)$ is the steady thermal problem solution. The equation (56) is numerically integrated in time by using the second order Crank-Nicolson scheme $\left(\varepsilon=\frac{1}{2}\right)$ which is formulated as follows:

$$
\frac{\theta^{n+1}-\theta^{n}}{\Delta t}=\varepsilon L_{\theta^{n+1}}(x, r, t)+(1-\varepsilon) L_{\theta^{n}}(x, r, t)
$$

where

$$
\begin{aligned}
L_{\theta}(x, r, t)= & -\frac{1}{h^{2}}\left(\frac{1}{r} \frac{\partial \psi}{\partial r} \cdot \frac{\partial \theta}{\partial x}-\frac{1}{r} \frac{\partial \psi}{\partial x} \cdot \frac{\partial \theta}{\partial r}+\frac{1}{r} \frac{\partial \varphi}{\partial r} A(t) \frac{\partial \theta}{\partial x}-\frac{1}{\widehat{\operatorname{Re} P r}} \Delta_{f} \theta\right) \\
& +\frac{1}{h^{2}}\left(\frac{1}{r} \frac{\partial \psi}{\partial x} \cdot \frac{\partial \theta_{R}}{\partial r}+\frac{1}{\left.\widehat{\operatorname{RePr}} \Delta_{f} \theta_{R}\right)}\right.
\end{aligned}
$$

By projecting (57) in the Galerkin basis $\left(q_{i}(x) p_{2 j}(r)\right)_{i j}$, one obtains at each time step a system of linear equations solved by the classical Gauss method.

One can notice that the use of Chebyshev polynomials in both axial and radial directions is not obvious, and contribute to emphasize this numerical method.

\section{Numerical resolution of the dynamic and energy problem using spectral collocation method}

\subsection{Numerical resolution of the dynamic problem}

For reasons of simplicity, we describe explicitly only the resolution of the steady dynamic problem. For the unsteady problem, we use Crank-Nicolson method for time integration as in (50); the unsteady problem resolution in space is identical to the steady case.

The main interest of collocation method compared with Galerkin formulation is its simplicity: it is not necessary to build a relevment function to take into account non homogeneous boundary conditions. We introduce these conditions directly in the matrix of the system and/or in the basis trial functions. For this reason, it is easy to compute collocation procedure. Let us explain this method for the steady dynamic problem. 
According to the general formulation of spectral methods (27), the stream-function $\tilde{\psi}$ is projected on trial functions in the same manner as equation (33):

$$
\tilde{\psi}(x, r)=\sum_{k=0}^{N_{x}} \sum_{l=0}^{N_{r}} \psi_{k l} P_{2 l}(r) Q_{k}(x)
$$

We can apply the same approach used in Galerkin method to determine trial functions $P_{2 l}(r)$ and $Q_{k}(x)$. All conditions given by (35-42) are available, except the second condition (36) for $P_{2 l}(r)$ and the second condition (41) for $Q_{k}(x)$. Then, with the method given by (34), we obtain:

$$
P_{2 l}(r)=T_{2 l}(r)+\frac{(l+2)^{2}-l^{2}}{(l+2)^{2}+(l+1)^{2}} T_{2(l+1)}(r)-\frac{(l+1)^{2}+l^{2}}{(l+2)^{2}+(l+1)^{2}} T_{2(l+2)}(r)
$$

and

$$
Q_{k}(x)=T_{k}(x)-\frac{k^{2}}{(k+2)^{2}} T_{k+2}(x)
$$

The vorticity function can be written as follows:

$$
\breve{\omega}=\sum_{k=0}^{N_{x}} \sum_{l=0}^{N_{r}} A_{k l}(x, r) \psi_{k l}
$$

where:

$$
\left\{\begin{array}{l}
A_{k l}(x, r)=A_{f} P_{l}(r) Q_{k}^{\prime \prime}(x)+B_{f} P_{l}^{\prime \prime}(r) Q_{k}(x)+C_{f} P_{l}^{\prime}(r) Q_{k}(x)+D_{f} P_{l}^{\prime}(r) Q_{k}^{\prime}(x) \\
A_{f}=h^{2} ; B_{f}=r^{2}+r^{2} h^{\prime 2} \quad ; \quad C_{f}=\rho\left(2 h^{\prime 2}-h h^{\prime \prime}\right)-\frac{\lambda^{2}}{r} ; D_{f}=-2 r h h^{\prime}
\end{array}\right.
$$

Then, substituting $\tilde{\psi}$ and $\breve{\omega}$ by their expressions (59), (62) in the steady dynamic equation (22), we obtain the following discretized dynamic equation:

$$
\begin{aligned}
& \frac{1}{r}\left[\left(\sum_{k=0}^{N_{x}} \sum_{l=0}^{N_{r}} P_{l}^{\prime}(r) Q_{k}(x) \psi_{k l}\right) \cdot\left(\sum_{k=0}^{N_{x}} \sum_{l=0}^{N_{r}} A_{k l}^{x}(x, r) \psi_{k l}\right)\right. \\
& \left.\quad-\left(\sum_{k=0}^{N_{x}} \sum_{l=0}^{N_{r}} P_{l}(r) Q_{k}^{\prime}(x) \psi_{k l}\right) \cdot\left(\sum_{k=0}^{N_{x}} \sum_{l=0}^{N_{r}} A_{k l}^{r}(x, r) \psi_{k l}\right)\right] \\
& +\frac{2}{r^{2}}\left[\left(\sum_{k=0}^{N_{x}} \sum_{l=0}^{N_{r}} P_{l}(r) Q_{k}^{\prime}(x) \psi_{k l}\right)-r \frac{h^{\prime}}{h}\left(\sum_{k=0}^{N_{x}} \sum_{l=0}^{N_{r}} P_{l}^{\prime}(r) Q_{k}(x) \psi_{k l}\right)\right]\left(\sum_{k=0}^{N_{x}} \sum_{l=0}^{N_{r}} A_{k l}(x, r) \psi_{k l}\right) \\
& =\frac{1}{\widehat{\operatorname{Re}}} \sum_{k=0}^{N_{x}} \sum_{1=0}^{N_{r}}\left[\begin{array}{l}
\left.A_{g}(x, r) A_{k l}^{x x}(x, r)+B_{g}(x, r) A_{k l}^{r r}(x, r)+C_{g}(x, r) A_{k l}^{r}(x, r)\right] \\
\left.+D_{g}(x, r) A_{k l}^{x r}(x, r)+E_{g}(x, r) A_{k l}^{r r}(x, r)+F_{g}(x, r) A_{k l}(x, r)\right]
\end{array}\right.
\end{aligned}
$$


with:

$$
A_{k l}^{\alpha}(x, r)=\frac{\partial A_{k l}(x, r)}{\partial \alpha} \text { and } A_{k l}^{\alpha \beta}(x, r)=\frac{\partial^{2} A_{k l}(x, r)}{\partial \alpha \partial \beta}
$$

where $\alpha$ or $\beta$ represents the space variable $x$ or $r$. The relation (65) is a function of local point $(x, r) \in \Delta$, where $\Delta$ is the square $[-1,1] \times[-1,1]$.

The collocation method consists to write the above equation on specific points $\left(x=x_{i}, r=r_{j}\right)_{\substack{0 \leq j \leq N_{r} \\ 0 \leq j \leq N_{x}}}$ of $\Delta$, called collocation points. We chose the collocation points of Chebyshev-Gauss-Lobatto [5], defined by:

$$
x_{i}=-\cos \left(i \pi / N_{x}\right) \text { and } r_{j}=-\cos \left(j \pi / N_{r}\right)
$$

with $0 \leq i \leq N_{x}$ and $0 \leq j \leq N_{r}$.

We recall that homogeneous boundary conditions are imposed on trial functions $P_{2 l}(r)$ and $Q_{k}(x)$. The non homogeneous boundary conditions, like Poiseuille profile at the duct entry or flow-rate condition at the wall, are directly imposed in the matrix system .

The nonlinear system obtained is solved by the Newton algorithm.

\subsection{Numerical resolution of the thermal problem}

Steady and unsteady energy equations are solved in the same manner as the dynamic problem, using spectral collocation method and Crank-Nicolson time-solver method described above.

Concerning trial functions for steady and unsteady thermal problems, we use directly Chebyshev polynomials:

$$
p_{l}(r)=T_{l}(r) \text { and } q_{k}(x)=T_{k}(x)
$$

All boundary conditions are imposed in the matrix system.

\section{Convective heat transfer}

The local convective heat transfer coefficient $h_{T}$ is written as follows:

$$
h_{T}(x, t)=\frac{\Phi_{W}}{\Delta T_{r e f}}
$$

where $\Delta T_{r e f}$ is a typical difference temperature reference. That one depends on the wall boundary conditions hypotheses. The main difficulty with convective unsteady heat transfer lies in the temperature reference choice. After several tests, we have chosen:

$$
\Delta T_{r e f}(x, t)=T_{W}-T_{m}(x, t)
$$

where $T_{m}(x, t)$ is the mean bulk temperature given by:

$$
T_{m}(x, t)=\frac{\int_{0}^{1} u(x, r, t) \cdot T(x, r, t) \cdot r d r}{\int_{0}^{1} u(x, r, t) \cdot r d r}
$$


The instantaneous convective heat transfer in unsteady flows can formally be defined by the local Nusselt number $N u(x, t)$, given by the relation:

$$
N u(x, t)=\frac{R h_{T}(x, t)}{k}
$$

With the variables transform (heading 4), the Nusselt number can be written as follows:

$$
N u(x, t)=\frac{1}{h \lambda} \sqrt{\lambda^{2}+h^{\prime 2}} \frac{\left(\frac{\partial \theta(x, t)}{\partial r}\right)_{W}}{1-\theta_{m}(x, t)}
$$

where $h^{\prime}$ is the derivative oh the function $h$.

\section{Numerical results}

\subsection{Definition of geometrical, physical and numerical parameters}

All results have been computed with Galerkin spectral method, except those used to make the comparison between Galerkin and collocation method (headings 6 and 7). The source of pulsations is located at the inlet section.The studied fluid is air, under normal conditions of temperature and pressure. The fluid flow is submitted to a pure sinusoidal pulsation. The previous studies $[1,2]$ showed that the numerical results are in the more stable mode if the ratio $R / L$ is small, compared to the unit. Consequently, the basic geometry parameters are:

$$
R=0,02 \mathrm{~m} ; L=0,08 \mathrm{~m} ; e=E / R=2, R_{V}=3 R=0,06 \mathrm{~m} .
$$

The sinusoidal surface of the wall is represented by the function $h$ :

$$
h(x)=1+\frac{e}{2}\left[1-\cos \left(\pi \cdot n_{O}(x+1)\right)\right]
$$

where $n_{O}$ indicates the number of geometrical periods chosen here equals to 3 .

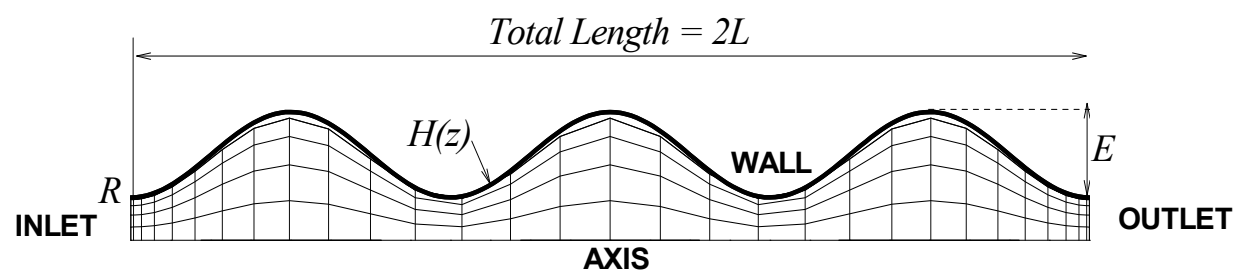

Fig. 1. Geometry of the study domain showing the projection of the Chebyshev -GaussLobatto mesh grid on the physical geometry $(\mathrm{Nx}=30, \mathrm{Nr}=5)$

\subsubsection{Choice for the orders of truncature}

To ensure the accuracy of our results from the numerical point of view, we try several orders of truncature in the Chebyshev basis developments (Batchi, 2005) for details. When the orders of truncature increase, let $e_{\alpha \beta}$ be the error calculated between two consecutive 
truncature orders $\alpha$ and $\beta$ of the stream function coefficients $\psi_{k l}$ (respectively the temperature coefficients $\theta_{k l}$ ) relative to the steady flow. The expression of $e_{\alpha \beta}$ is:

$$
e_{\alpha \beta}=\max _{k, l}\left|f_{k l}^{\alpha}-f_{k l}^{\beta}\right|
$$

where $f^{\alpha}$ represents $\psi$ or $\theta$, for the truncature order $\alpha$.

For the dynamic point of view, we note first that the truncature errors $e_{\alpha \beta}$ depend mainly on the parameter $N x$. This means that the increase in the number of polynomials in the radial direction does not improve the convergence of the results. Secondly, figure 2.a shows that, globally, the amplitudes of $e_{\alpha \beta}$ decrease when the values of $N x$ increase. With $N r$ fixed to 5 and $N x \geq 30$, the truncature errors $e_{\alpha \beta}$ are negligible, about $2.10^{-4}$.

For a given value of $M r$, we observe in figure $2 . \mathrm{b}$ a good convergence of the temperature coefficients when $M x$ increases. But, unlike dynamic field, for the range of $M r$ values between 5 and 9, the analysis of the thermal field leads to slightly different conclusions. Indeed, probably due to the temperature conditions imposed on the entry section, the thermal field is more sensitive to the parameter $M r$ than dynamic field. For a fixed value of $M x$, the temperature truncature errors increase with $M r$. Then, optimal convergence is obtained for $M r=5$. For this value, the truncature error is less than $10^{-8}$ when $M x>56$. In conclusion, we have selected for the dynamic problem: $N x=30$ and $N r=5$, and for the thermal problem, we have chosen: $M x=120$ and $M r=5$.

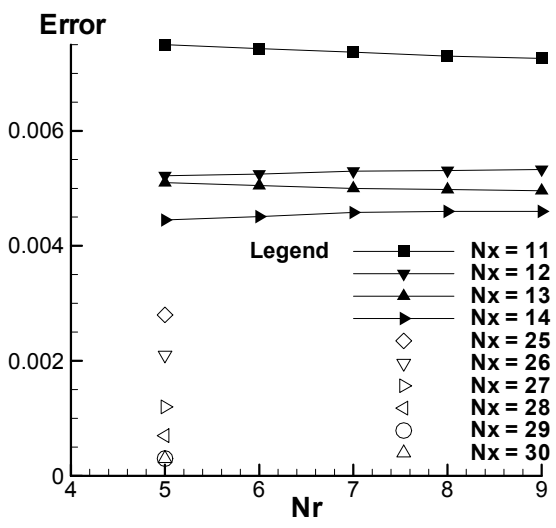

(a)

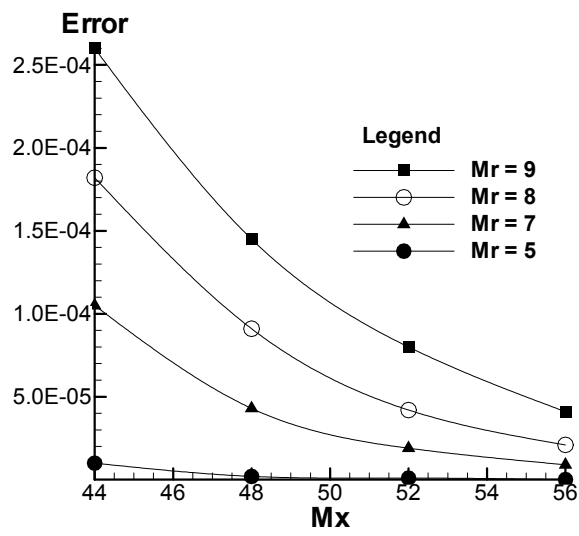

(b)

Fig. 2. a) Maximum truncature error in the Chebyshev basis development of the stream function $\psi$ (steady flow, $\operatorname{Re}=30$ ). b) Maximum truncature error in the Chebyshev basis development of the temperature function $\theta$ (steady flow, $\operatorname{Re}=30, \operatorname{Pr}=0.73$ )

\subsection{The steady flow}

\subsubsection{Study of the dynamic field}

In order to study the dynamic behaviour of the flow according to the flow-rate, we varied the Reynolds number from 1 to 50. Figure 3 shows that the flow remains "with parallel lines", i.e. of crawling type, until $\mathrm{Re}=10$. From this value, a vortex initially appears in the 
first geometrical period, with a center shifted upstream and close to the wall. Then, when Re increases, a less bulky vortex appears in the two other geometrical periods. The center of each vortex moves towards the downstream while moving away from the wall more and more gradually. These results perfectly agree with those previously shown by Blancher, 1991; Batina et al., 2004, 2009.
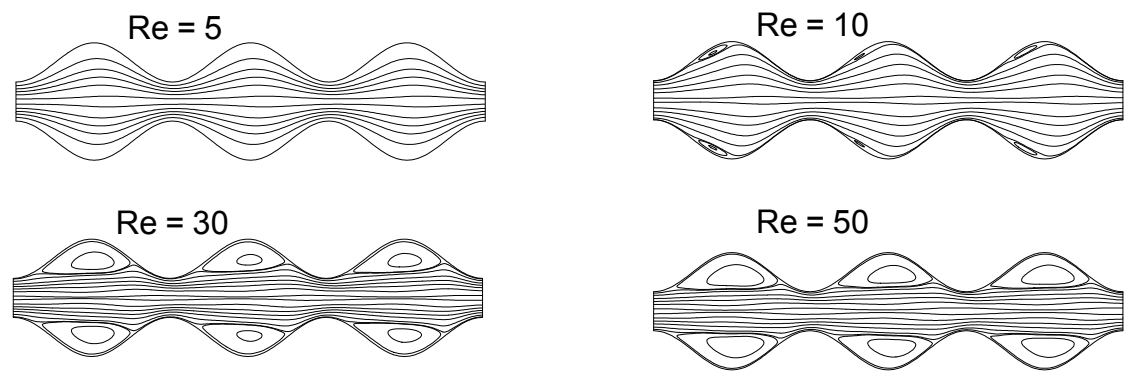

Fig. 3. Streamlines parametric study versus Reynolds number (steady flow)

\subsubsection{Thermal study}

Figure 4 shows a comparative study of the convective heat transfer by means of the Nusselt number, in stationary regime. One can clearly see that the vortex has a negative influence on the heat transfer on almost the totality of the duct, except for the entry. Locally, we observe a light heat transfer enhancement at the constriction which increases with the amplitude of geometry.

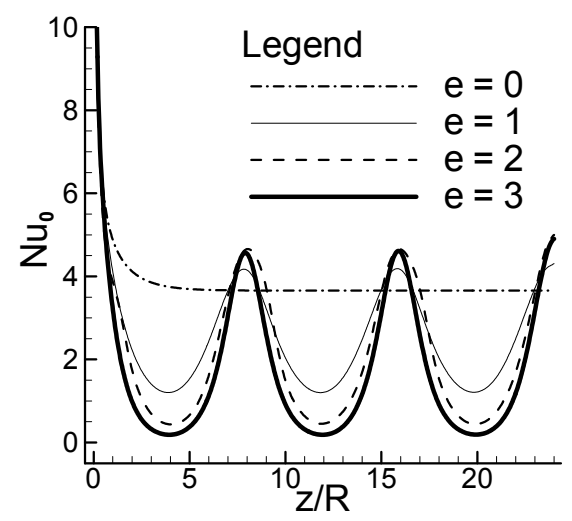

Fig. 4. Heat transfer comparison (steady case): parametric study according to the reduced amplitude e of the geometry

\subsection{The unsteady flow}

In order to maintain the bidimensional hypothesis, the flow is submitted to low frequencies $(0 \leq f \leq 5 \mathrm{~Hz})$ and the amplitude of pulsation $\tau$ do not exceed 0.7. The number of time steps 
by period is equal to 24 . The Reynolds number is fixed to 30 corresponding to a total filling of the furrows. The corresponding steady regime is taken as initial condition for the unsteady mode (instant $t=0$ ).

To understand better the fluid dynamic behaviour in pulsed regime, figure 5 shows the detail of the streamlines for one period $T$. We note that the vortices quickly disappear during the first instants, from $t=0$ to $t=2 T / 8$. This interval of time corresponds to the phase of the flow acceleration, with a maximum reached for $t=2 T / 8$. After that, a phase of deceleration appears, with a passage to zero for $t=T / 2$. The size of the vortex is maximal for $t=6 \mathrm{~T} / 8$. This stage corresponds to the maximum of the flow deceleration $(\Omega t=3 \pi / 2)$. In the central zone, the flow moves in positive direction, and close to the wall, the flow moves in opposite direction. After this, the fluid moves more closer to the wall. For the acceleration phase which follows, the flow tends to take its initial aspect again. However, with $t=T$, we approximately find the form of the flow for $t=0$.
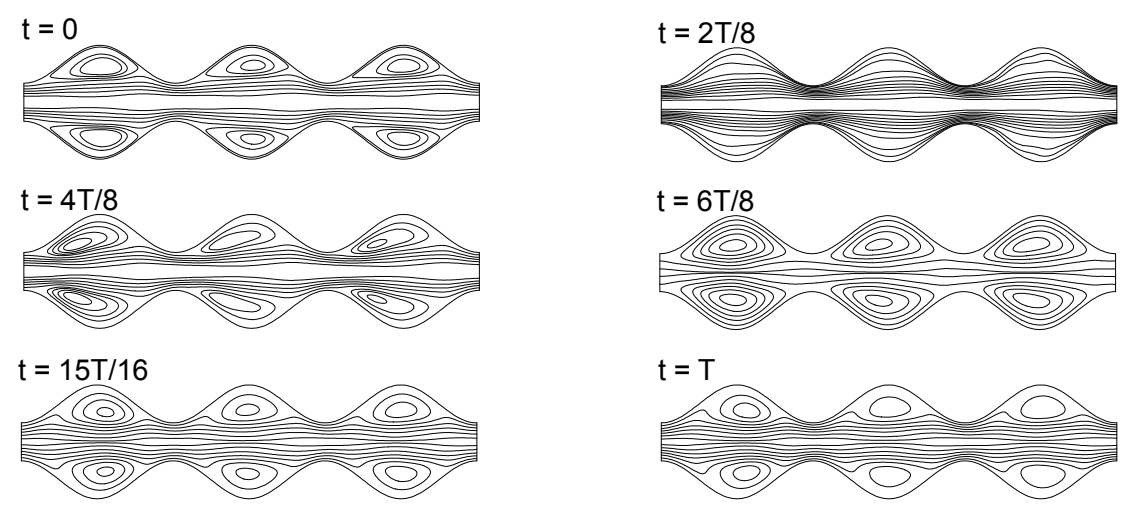

Fig. 5. Time history streamlines during one period, $\Omega=0.3, \operatorname{Re}=30, \operatorname{Pr}=0.73, \tau=0.7$

\subsubsection{Temporal evolution of the unsteady temperature field}

Let us locate first particular control points in the duct (figure 6). Each point is chosen because we expect significant results on dynamical and thermal phenomena close to this region.

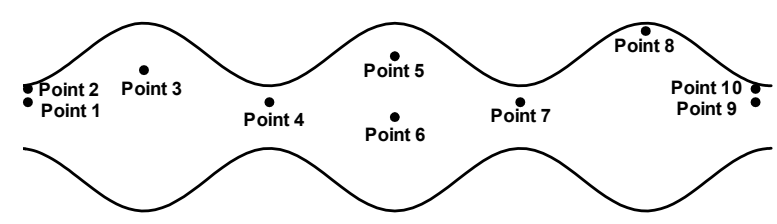

Fig. 6. Localization of control points for the description of the time-history phenomena

In order to have a global vision of the dynamic and thermal unsteady phenomena, we carried out a spectral analysis with the FFT method, for the velocity and temperature fields, on three temporal periods $(\mathrm{t}>10)$. The figures $7 . \mathrm{a}$ and $7 . \mathrm{b}$ show that the most significant 
dynamic fluctuations are located at each constriction of the tube for axial velocities and downstream the constriction for radial velocities.
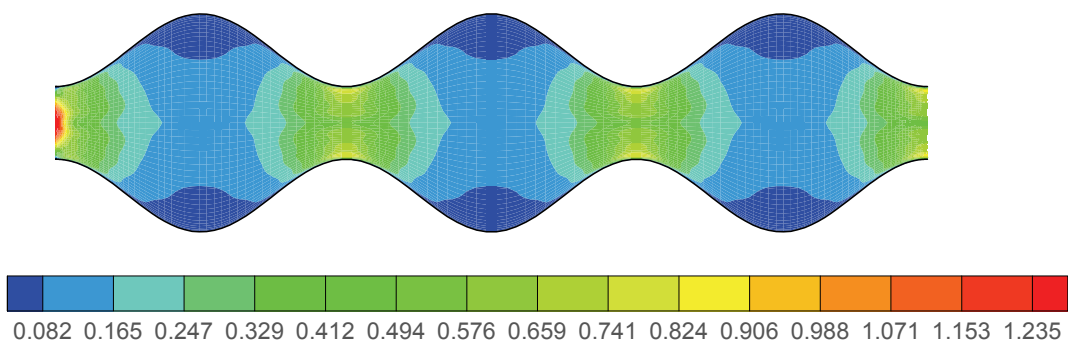

(a)
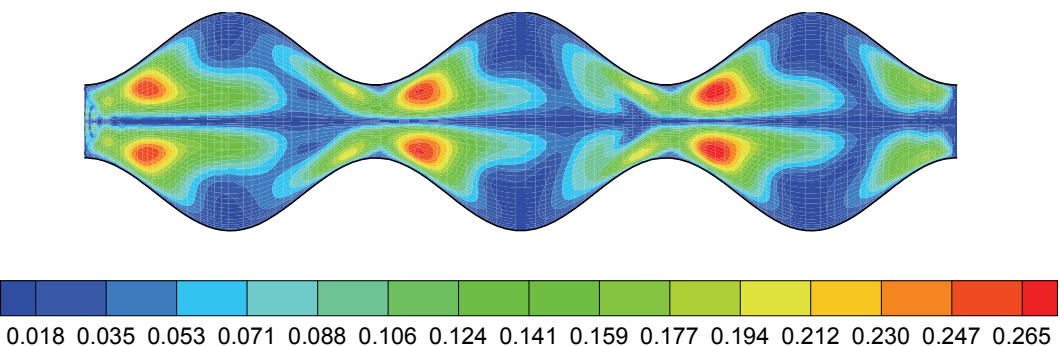

(b)
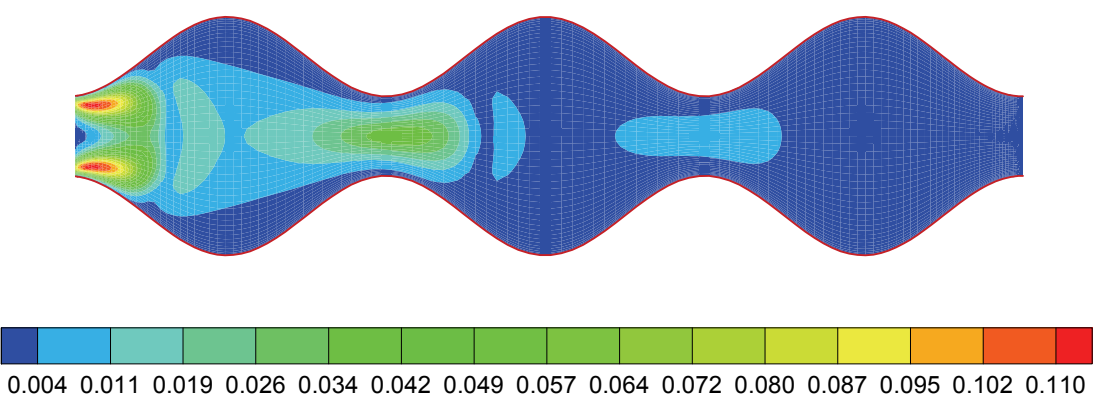

(c)

Fig. 7. Amplitudes fluctuations of the axial velocity (a), the radial velocity (b) and the temperature (c). $\operatorname{Re}=30, \operatorname{Pr}=0.73, \tau=0.7$

One can thus expect a substantial modification of the thermal convective heat transfer in these privileged areas, due to the thermal boundary modifications corresponding to the entry section duct, and in the minimum sections as shown in figure 7.c. 


\subsection{Unsteady convective heat transfer}

On figure 8 , we study, on the control point 4, the Nusselt number evolution versus the pulsation frequency $\Omega$. This amplitude analysis is obtained by the FFT method realised on the instantaneous Nusselt number defined by equation (71). We observe the decrease of the Nusselt number amplitudes when $\Omega$ increases.

The instantaneous heat transfer does not correspond to a measurable physical reality. Thus it is necessary to consider the time averaged Nusselt number. So, we define:

$$
\overline{N u}(x)=\langle N u(x, t)\rangle=\frac{\omega}{2 \pi} \int_{0}^{\frac{2 \pi}{\omega}} N u(x, t) d t
$$

To evaluate the contribution of the pulsation on the heat transfer, we compare $\overline{N u}(x)$ with the Nusselt number $N u_{0}(x)$ obtained in steady flow. We confirm in figure 9, a very significant increase of the heat transfer located at the constriction, and conversely a high reduction at maximum radius areas (zones of dead fluid).

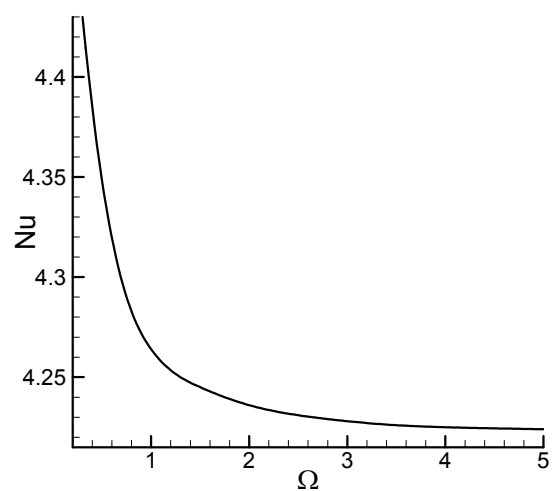

Fig. 8. Evolution of Nusselt number (FFT method) versus the pulsation frequency on the control point $4(\operatorname{Re}=30 ; \operatorname{Pr}=0.73 ; \tau=0.7)$

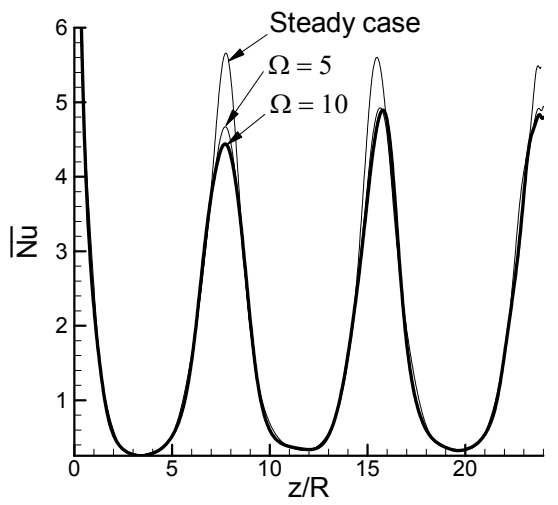

Fig. 9. Heat transfer comparison in steady and unsteady flow $(\Omega=10, \Omega=5, \tau=0.7$ ) 


\subsection{Comparison between Galerkin and collocation spectral methods 9.5.1 Dynamic and thermal results comparison}

In order to make comparison between Galerkin and collocation spectral methods, classical parameters are chosen: $\mathrm{Re}=30, \mathrm{Nx}=30$ and $\mathrm{Nr}=5$ for both methods.

When the flow is pulsed, we chose to study the dynamic and thermal fields at points $1,7,8$ and 9 of figure 6. Apart the periodicity previously highlighted, these curves show once again that there is nearly no difference between the two methods as shown in figures 10 .

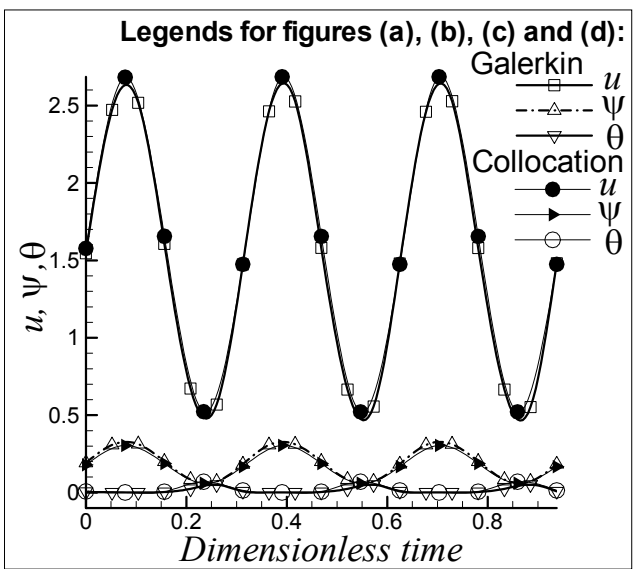

(a)

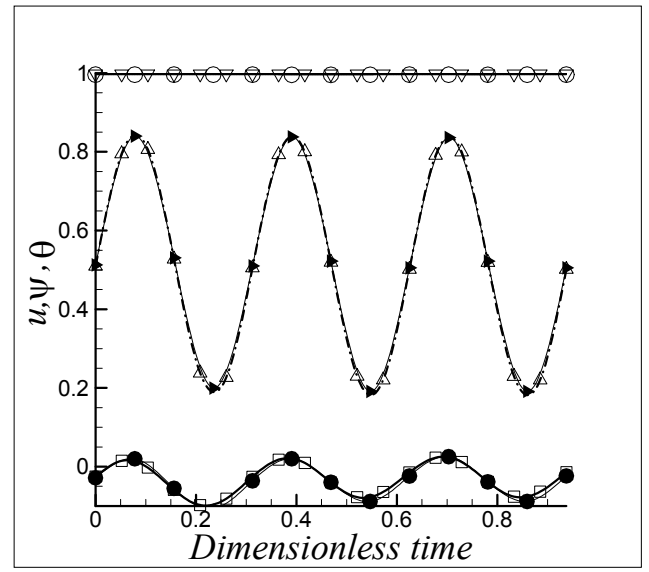

(c)

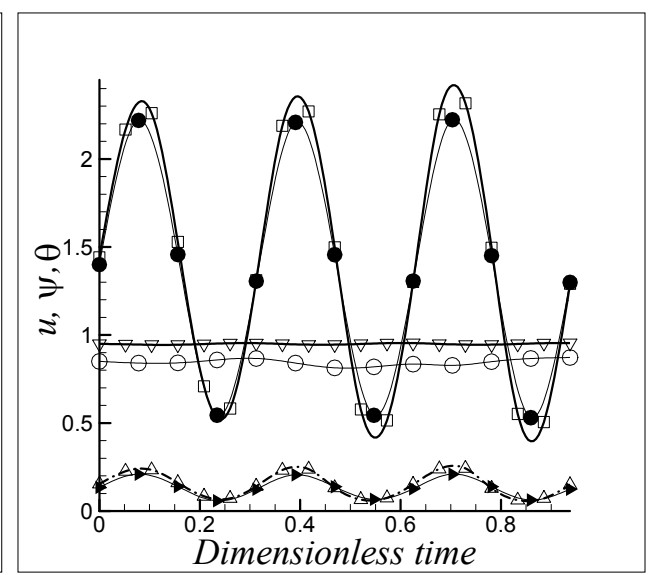

(b)

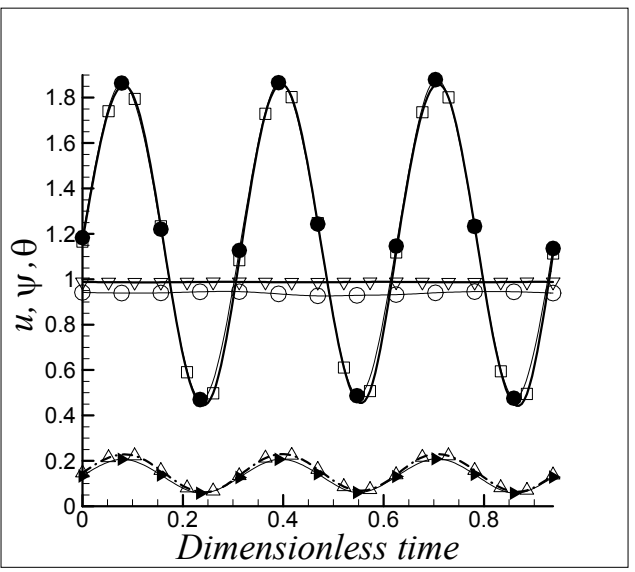

(d)

Fig. 10. Comparison between Galerkin and collocation methods during 3 periods, for control points: (a): point 1 ; (b): point 7; (c): point 8 ; (d): point 9. $(\Omega=0.3, \operatorname{Re}=30, \operatorname{Pr}=0.73, \tau=0.7)$

In steady flow, the longitudinal evolution of heat transfer characterized by the Nusselt number shows a slight difference between the two models, located particularly in the vicinity of geometry furrows and constrictions (figure 11.a.). However, this difference does 
not exceed two or three percent. In terms of unsteady heat transfer, comparison between the two methods shows that the average Nusselt number $\overline{N u}(x)$ given by equation (75) presents slight differences similar to those observed in steady state regime (figure 11.b.).

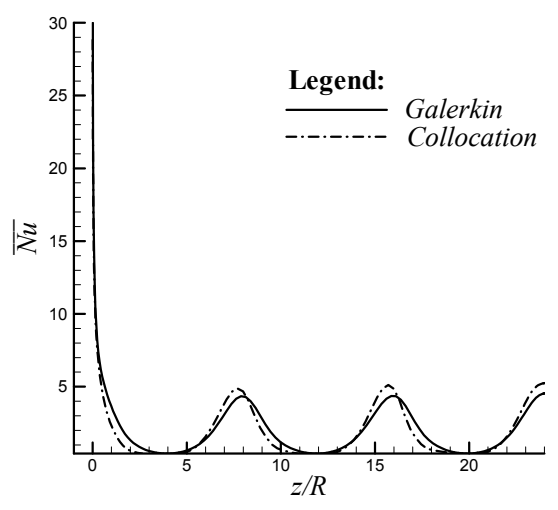

(a)

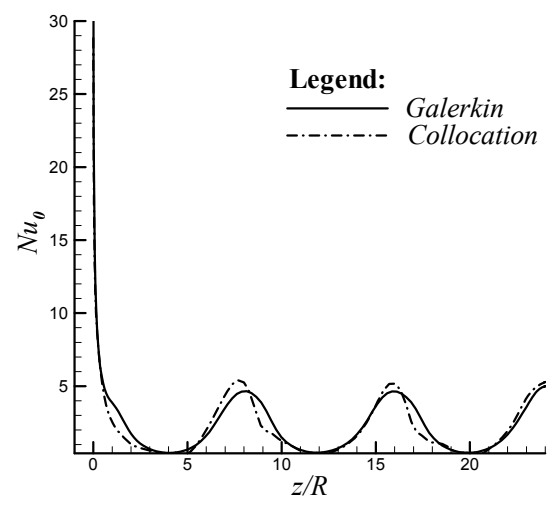

(b)

Fig. 11. Heat transfer comparison between Galerkin and collocation methods. (a): steady case; (b): unsteady case $(\Omega=0.3, \operatorname{Re}=30, \operatorname{Pr}=0.73, \tau=0.7)$

\subsubsection{Comparison of performances and speed computations}

We have shown that Galerkin and collocation spectral methods, developed to the same truncature orders, give results with similar accuracy. The essential difference between these methods lies in their performances, rapidity and simplicity of computational implementation. The table below summarizes their performances. Let us define before:

- On the one hand: $C P U_{0 G}$ and $C P U_{0 C}$ the CPU time to obtain the steady regime with Galerkin and collocation method respectively; $C P U_{1 G}$ and $C P U_{1 C}$ the CPU time to compute one period of the unsteady regime with Galerkin and collocation method respectively;

- On the other hand: Newton 0 and Newton $_{0 C}$ the number of Newton iterations to compute the steady regime with Galerkin and collocation method respectively; Newton $_{1 G}$ and Newton $1 C$ the number of Newton iterations to compute one time step with Galerkin and collocation method respectively.

At last, we define the ratios:

$$
C P U_{0}=\frac{C P U_{0 G}}{C P U_{0 C}} ; C P U_{1}=\frac{C P U_{1 G}}{C P U_{1 C}} ; \text { Newton }_{0}=\frac{\text { Newton }_{0 G}}{\text { Newton }_{0 C}} ; \text { Newton }_{1}=\frac{\text { Newton }_{1 G}}{\text { Newton }_{1 C}}
$$

Then, we obtain the following results:, performed on the same computer:

\begin{tabular}{|l|l|l|l|}
\hline $\mathrm{CPU}_{0}$ & $\mathrm{CPU}_{1}$ & Newton $_{0}$ & Newton $_{1}$ \\
\hline 2.4 & 5.2 & 3.2 & 3.2 \\
\hline
\end{tabular}

Table 1. Comparison of performances between Galerkin and collocation spectral methods 


\section{Conclusions}

In this paper, numerical studies have been carried out on pulsating flows through axisymmetric sinusoidal ducts. Thus, the study emphasizes on the heat transfer modifications in this particular flows with rates modulation close to the unit. The results obtained have shown that the flow is influenced by many parameters including Reynolds numbers, rate modulation and amplitude of geometry. We observe that the results are encouraging and offer good perspectives in pulsed internal flows cases. From numerical point of view, the results obtained confirm the previous general conclusions in axisymmetric geometries (André et al., 1987, 1981; Batina et al., 1989, 1991, 2009), i.e.:

- For the steady regime: all classical results are obtained with high precision.

- For the unsteady regime, dynamic and thermal fields show an important heat transfer enhancement in the entry zone. A dynamic and thermal shock occur nearly this area. Mechanical tube behaviour can be modified in this region and the shear stress occurring during the pulsation can induce some damage if the tube is connected to a big tank. This phenomenon is of great interest in industrial structures. Nevertheless, convective heat transfer decrease when the fluid moves forward in the tube.

The numerical solution was performed using Galerkin and collocation spectral methods whose main features were recalled. The numerical results obtained show that:

- Steady and unsteady regimes do not depend on the spectral method used;

- The unsteady dynamic and thermal phenomena are periodic in time, with a frequency equal to the frequency of pulsations.

However, the collocation method is simpler to use and its computer calculations are faster.

Out of these general conclusions, this study focusing on sinusoidal geometries induce especially zones of dead fluid that locally have a negative influence on heat transfer, particularly for the steady flow. The spatial periodicity of the steady flow in this type of geometry is acquired only at the end of the second, even third geometrical period. The transient phenomena are therefore relatively short in time. Thus, the dynamic and thermal flow behaviours become periodic.

Compared to models based on classical methods such as asymptotic developments, finite differences, finite volumes, finite elements, etc, our numerical method leads to the following remarks:

- The accuracy of the present model is high.

- The present computational code is easier to build compared to finite elements one, for example.

- If we consider the CPU time, the present model needs few minutes to compute the numerical equations. This result traduces the efficiency of our model which is easier and more adapted to solve this particular problem. Nevertheless, compared to some industrial codes, our model have some disadvantages, such as:

- $\quad$ the non linear coupled unsteady terms in Navier Stokes and Energy equations are not taken into account in the present model

- $\quad$ this problem require smoother geometries

- the order of polynomials developments increases strongly the computing time

- when the modulation flow-rate approaches or exceeds the unity, we must choose carefully some data to assume the algorithms convergence. For example, when $\tau>100 \%$, convergence conditions impose small time steps, and the CPU time on classical computers can make our code prohibitive. 
Actually, our model is extended to non linear coupled problems of unsteady Navier Stokes and Energy equations, and the results are very encouraging. Moreover, our model treats successfully the problem of heat and mass transfer in natural convection.

In a final analysis, our numerical method based on a suitable spectral method is of a good accuracy. One of its originality is the choice of Chebyshev polynomials basis in both axial and radial directions, and the use of a shift operator technique allow the introduction of non homogeneous boundary conditions in Galerkin formulation. The automatic construction of these polynomials basis is of a great interest. These particular mathematical and numerical tools have permitted the resolution of this non obvious problem which consists on pulsated unsteady flows associated to simultaneous developments dynamic and thermal fields.

\section{References}

André, P.; Creff, R. \& Batina, J. (1981). Etude des conditions particulières de fréquence favorisant les transferts thermiques en écoulements pulsés en canalisation cylindrique, International Journal of Heat and Mass Transfer, Vol. 24, pp. 1211-1219.

André, P.; Creff, R. \& Batina, J. (1987). Study of thermal fluid for pulsed flow with compressible fluids, Numerical Methods in Thermal Problems., Fifth international conference., Montreal, Canada, Pineridge Press, pp. 149-154, 1987.

Batchi, M. (2005). Etude Mathématique et Numérique des Phénomènes de Transferts Thermiques liés aux Ecoulements Instationnaires en Géométrie Axisymétrique, Thèse de Doctorat Mathématiques Appliquées, Université de Pau.

Batina, J.; Creff, R.; André, P. \& Blancher, S. (1989). Numerical model for dynamic and thermal developments of a turbulent pulsed ducted flow, Proceedings Eurotherm, n9, pp. 50-57, Bochum, Germany.

Batina, J.; André, P.; Creff, R. \& Blancher, S. (1991). Dynamic and thermal developments of a pulsed laminar compressible ducted flow, Proceedigs Eurotherm, n²5, pp. 227-234, Pau, France.

Batina, J. (1995). Etude numérique des écoulements instationnaires pulsés en canalisation cylindrique, Thèse de Doctorat Physique, Université de Pau.

Batina, J.; Creff, R. \& Batchi, M. (2004). Etude thermique convective d'un écoulement interne en géométrie axisymétrique sinusoïdale, Actes du congrès SFT 04, pp. 987-992.

Batina, J.; Batchi, M.; Blancher, S.; Creff, R. \& Amrouche, C. (2009). Convective heat transfer augmentation through vortex shedding in sinusoidal constricted tube., International Journal of Numerical Methods for Heat and Fluid Flow, Vol.19, N.3/4, pp. 374-395.

Bernardi, C. \& Maday, Y. (1992). Approximations spectrales de problèmes aux limites elliptiques, Springer-Verlag.

Blancher, S. (1991). Transfert convectif stationnaire et stabilité hydrodynamique en géométrie périodique, Thèse de Doctorat Physique, Université de Pau.

Canuto, C.; Hussaini, M.Y.; Quarteroni, A. \& Zang, T.A. (1988). Spectral methods in fluids dynamics, Springer-Verlag.

Chakravarty, S. \& Sannigrahi A.K. (1999). A nonlinear mathematical model of blood flow in a constricted artery experiencing body acceleration, Mathematical and Computer Modeling, vol. 29, pp. 9-25.

Creff, R.; André, P. \& Batina, J. (1985). Dynamic and Convective Results for a Developping Laminar Unsteady Flow, International Journal of Numerical Methods in Fluids, Vol. 5, pp. 745-760. 
Ghaddar, N.K.; Magen, M.; Mikic, B.B. \& Patera, A. (1986). Numerical investigation of incompressible flow in grooved channels, Resonance and oscillatory heat transfer enhancement, Journal of Fluid Mechanics, vol.168, pp. 541-567.

Gelfgat, A.Y. (2004). Stability and slightly supercritical oscillatory regimes of natural convection in a 8:1 cavity: solution of the benchmark problem by a global Galerkin method, International Journal of Numerical Methods in Fluids, vol. 44, pp. 135-146.

Hemida, H.N.; Sabry, M.N.; Abdel-Rahim, A. \& Mansour, H. (2002). Theoretical analysis of heat transfer in laminar pulsating flow, International Journal of Heat and Mass Transfer, vol. 45, pp. 1767-178.

Lee, B.S.; Kang, L.S. \& Lim, H.C. (1999). Chaotic mixing and mass transfer enhancement by pulsatile laminar flow in an axisymmetric wavy channel, International Journal of Heat and Mass Transfer, Vol. 42, pp. 2571-2581.

Moschandreau, T. \& Zamir, M. (1997). Heat transfer in a tube with pulsating flow and constant heat flux, International Journal of Heat and Mass Transfer, vol.. 40, n¹0, pp. 2461-2466.

Shen, J. (1994). Efficient spectral-Galerkin method I: direct solvers for the second and fourth order equations using Legendre polynomials, SIAM J. Sci. Comput, vol.15, nº, pp. 1489-1505.

Shen, J. (1995). Efficient spectral-Galerkin methods II: direct solvers of second and fourth order equations by using Chebyshev polynomials, SIAM J. Sci. Comput, vol.16, ${ }^{\circ} 8$, pp. 74-87.

Shen, J. (1997). Efficient spectral-Galerkin methods III: polar and cylindrical geometries, SIAM J. Sci. Comput, vol.18, n6, 1583-1604.

Sobey, I.J. (1980). On flow through channels. Part 1: Calculated flow patterns, Journal of Fluid Mechanics, vol. 96, pp. 1-26.

Young Kim, S.; Ha Kang, B. \& Min Hyun, J. (1998). Forced convection heat transfer from two heated blocks in pulsating channel flow, International Journal of Heat and Mass Transfer, vol. 41, n³, pp. 625-634. 


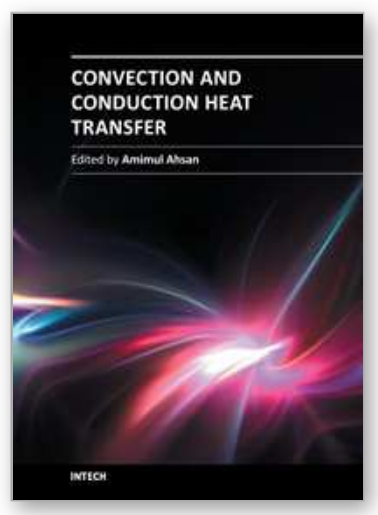

\section{Convection and Conduction Heat Transfer}

Edited by Dr. Amimul Ahsan

ISBN 978-953-307-582-2

Hard cover, 394 pages

Publisher InTech

Published online 17, October, 2011

Published in print edition October, 2011

The convection and conduction heat transfer, thermal conductivity, and phase transformations are significant issues in a design of wide range of industrial processes and devices. This book includes 18 advanced and revised contributions, and it covers mainly (1) heat convection, (2) heat conduction, and (3) heat transfer analysis. The first section introduces mixed convection studies on inclined channels, double diffusive coupling, and on lid driven trapezoidal cavity, forced natural convection through a roof, convection on non-isothermal jet oscillations, unsteady pulsed flow, and hydromagnetic flow with thermal radiation. The second section covers heat conduction in capillary porous bodies and in structures made of functionally graded materials, integral transforms for heat conduction problems, non-linear radiative-conductive heat transfer, thermal conductivity of gas diffusion layers and multi-component natural systems, thermal behavior of the ink, primer and paint, heating in biothermal systems, and RBF finite difference approach in heat conduction. The third section includes heat transfer analysis of reinforced concrete beam, modeling of heat transfer and phase transformations, boundary conditions-surface heat flux and temperature, simulation of phase change materials, and finite element methods of factorial design. The advanced idea and information described here will be fruitful for the readers to find a sustainable solution in an industrialized society.

\section{How to reference}

In order to correctly reference this scholarly work, feel free to copy and paste the following:

J. Batina, S. Blancher, C. Amrouche, M. Batchi and R. Creff (2011). Convective Heat Transfer of Unsteady Pulsed Flow in Sinusoidal Constricted Tube, Convection and Conduction Heat Transfer, Dr. Amimul Ahsan (Ed.), ISBN: 978-953-307-582-2, InTech, Available from: http://www.intechopen.com/books/convection-andconduction-heat-transfer/convective-heat-transfer-of-unsteady-pulsed-flow-in-sinusoidal-constricted-tube

\section{INTECH}

open science | open minds

\author{
InTech Europe \\ University Campus STeP Ri \\ Slavka Krautzeka 83/A \\ 51000 Rijeka, Croatia \\ Phone: +385 (51) 770447 \\ Fax: +385 (51) 686166 \\ www.intechopen.com
}

\author{
InTech China \\ Unit 405, Office Block, Hotel Equatorial Shanghai \\ No.65, Yan An Road (West), Shanghai, 200040, China \\ 中国上海市延安西路65号上海国际贵都大饭店办公楼 405 单元 \\ Phone: +86-21-62489820 \\ Fax: +86-21-62489821
}


(C) 2011 The Author(s). Licensee IntechOpen. This is an open access article distributed under the terms of the Creative Commons Attribution 3.0 License, which permits unrestricted use, distribution, and reproduction in any medium, provided the original work is properly cited. 